\title{
Effects of Functional Group Interactions on the Gas-Phase Methylation and Dissociation of Acids and Esters
}

\author{
John J. Isbell and Jennifer S. Brodbelt \\ Department of Chemistry and Biochemistry, The University of Texas at Austin, Mustin, Texas, USA
}

\begin{abstract}
Functional group interactions have been observed to affect gas-phase ion-molecule chemistry in a quadrupole ion trap mass spectrometer. Gas-phase metlylation and collisionactivated dissociation reactions of a series of related acids and esters allows an evaluation of the structural factors that influence reactivity and functional group interactions of these compounds. Examination of the $[\mathrm{M}+\mathrm{H}]^{+}$or $[\mathrm{M}+15]^{+}$product ions by collision-activated dissociation has provided insight into the conformations from which diacids and diesters undergo electrophilic addition. Collision-activated dissociation has provided not only more detailed information on the structures of the ions, but also the data necessary for confident mechanistic interpretation. Iabeling studies were done to probe fragmentation pathways. Upon activation of the $\left[\mathrm{M}+\mathrm{CD}_{3}\right]^{+}$products of dimethyl maleate and dimethyl succinate, formed from reaction of the neutrals with $\mathrm{CD}_{3} \mathrm{OCD}_{2}^{+}$ions, a rapid interfunctional group methyl transfer causes scrambling of the methyls prior to elimination of dimethyl ether or methanol. The $[\mathrm{M}+15]^{+}$ions of dimethyl maleate are believed to lose dimethyl ether through a rate-determining 1,6-methyl transfer, whereas the $[M+15]^{+}$ions of dimethyl succinate eliminate methanol through a rate-determining 1,5-proton transfer. ( $\mathrm{J}$ Am Soc Mass Spectrom $1996,7,565-572)$
\end{abstract}

$\mathrm{F}$ unctional group interactions are among the most important classes of substituent effects in both solution and gas-phase chemistry [1-18]. The term "intramolecular catalysis" has bcen used to describe those types of functional group interactions that facilitate specific reactions or dissociation channels [17]. Such effects have been observed for a variety of difunctional molecules, which include diols and amino alcohols $[3-7,18 \mathrm{c}]$.

Some of the most striking functional group interactions have been observed for dicarboxylic acids and esters. For instance the ability of a functional group to interact with another can be correlated with distinctive fragmentation patterns upon electron ionization [1] or with the extent of dehydration of the diacids upon protonation by chemical ionization (CI) [9]. Similar results have been reported for related esters [13, 14, 19, 20] and fatty acids [2]. Extensive work has been done via electron ionization to examine functional group interactions [17]. For example, by using 2, 3-dideutero maleic and fumaric acids, Benoit et al. [1] observed the former decarboxylates after an intramolecular hydrogen transfer and the resulting ion can dehydrate, and lose water- $d_{n}$. The latter isomer exhibited an abundant

Address reprint requests to Professor Jennifer S. Brodbelt, Depart ment of Chemistry and Biochemistry, University of Texas at Austin. Austin, TX 78712-116?. molecular ion and sizcable losses of $\mathrm{H}_{2} \mathrm{O}$ and HDO. There was no $\mathrm{H} / \mathrm{D}$ scrambling for either diacid and these losses were postulated to occur via different pathways. Tajima et al. [15] found that the molecular ions of both dimethyl maleate and fumarate lose methoxy radical via a direct cleavage, and the latter molecular ion also rearranged to lose formaldehyde and $\mathrm{CH}_{3} \mathrm{CO}$ radical. Labeling experiments showed no kinetic isotope effect for the direct cleavage, but the rearrangement seen for the fumarate had an inverse isotope effect, which was attribuled to a rate-determining cyclization.

The chemistry of these acid ions changes dramatically when open-shell species formed by electron ionization are compared to the closed-shell ions formed from protonation upon chemical ionization. For example, Harrison et al. [9] found that under methane or hydrogen chemical ionization conditions, protonated Z-diacids eliminated water preferentially, whereas the protonaled $L$-diacids remained intact. The rationale for this result was attributed to the ability of the Z-diacid to donate a proton to the other functional group prior to dehydration and, second, the stability of the resulting product ion. Under the same $\mathrm{CI}$ conditions, diethyl fumarate formed predominantly the intact $[\mathrm{M}+\mathrm{H}]^{+}$ ion, whereas its $Z$-isomer lost ethanol from the analogous ion [9]. The argument Harrison put forth in this study agreed with earlier work [14] in which a series of variable alkyl chain diacids and the corresponding 
dimethyl esters exhibited, on protonation under methane $\mathrm{CI}$, increased dehydration or methanol elimination with increasing chain length. In contrast, by using an alternate ionizing technique-fast-atom bombardment - the protonated maleic acid showed less dehydration than the $[\mathrm{M}+\mathrm{H}]^{+}$of fumaric acid, which also yielded those products that result from subsequent decarbonylation and from decarboxylation [16]. The reduced degree of fragmentation observed for the $Z$-acids relative to their $E$-isomers was attributed to the ability for intramolecular hydrogen-bond formation.

$\Lambda$ concern in these types of studies has been the possibility of isomer interconversion in the course of the experiments. A charge-exchange study done by Harrison et al. [19] presented results that demonstrated that the molecular ions of diethyl maleate and fumarate do not interconvert at high internal energies, but that they do at low internal energies. However, Weisz et al. [20] examined the products of collisional activation of the protonated esters in a triple stage quadrupole mass spectrometer and found that protonated diethyl maleate exhibited $[\mathrm{M}+\mathrm{H}-\mathrm{EtOH}]^{+}$ and $\left[\mathrm{M}+\mathrm{H}-\mathrm{EtOH}-\mathrm{C}_{2} \mathrm{H}_{4}\right]^{+}$fragment ions, whereas protonated diethyl fumarate lost one or two elhylene units. Thus, low energy collision-activated dissociation (CAD) of the protonated diethyl esters of fumaric and maleic acids resulted in stereospecific dissociation pathways, which thereby demonstrated that these two isomers did not interconvert on collisional activation.

The primary goal of our systematic study of functional group interactions is to gain a better understanding of structure-reactivity relationships in gas-phase reactivity and fragmentation pathways of resulting ion-molecule reaction products. For example study of the CAD spectra of diacid ions and their corresponding methyl ester ions enables the effect of methyl substitution on reactivity to be determined and the extent of functional group interactions to be evaluated. Such sludies can lead to a better understanding of the factors that mediate ion-molecule reaction products, pathways, and reactivitics. Because functional group interaction is central to the success of many reactions in biochemical systems, these mass spectrometric investigations may provide new insight into understanding the intrinsic reactivities of complex substrates. The main objective of the present work is to determine the effects on the reactivity of the acid and ester functionalities toward dimethyl ether ions $[4-7,21,22]$ as a function of conjugation, the presence of electronwithdrawing groups, and functional group interactions, such as hydrogen bonding. Dimethyl ether was chosen as a selective chemical ionization reagent because it has proven capabilities as a probe of functional group interactions $[4-7,21,22]$. Dimethyl ether forms two reactive species: protonated dimethyl ether $(\mathrm{m} / z$ $47)$ and methoxymethylene $(\mathrm{m} / z$ 45). The former ion is able to transfer a proton, provided the gas-phase basic- ity of the analyte exceeds that of dimethyl ether. The latter can either methylate or effect a methylene substitution. Earlier work on amino alcohols has shown the $[\mathrm{M}+15]^{+}$product to result from an $\mathrm{S}_{\mathrm{N}} 2$ reaction, whereas the $[M+13]^{+}$stems from an additionelimination reaction, and this latter reaction was found to require the presence of a labile proton [21]. In this report, the CAD spectra of the $\left[\mathrm{M}+\mathrm{H}^{+}\right.$ions and the $[\mathrm{M}+15]^{+}$ions are examined with the aim of determining the reactive conformers and elucidating the mechanisms of fragmentation.

\section{Experimental}

A Finnigan quadrupole ion trap mass spectrometer (ITMS; Finnigan-MAT, San Jose, CA) was used in this study. The chamber was heated to $393 \mathrm{~K}$ and all liquid samples were introduced through heated leak valves at a nominal pressure of $1-2 \times 10^{-6}$ torr (uncorrected). Solids were introduced via a heated direct insertion probe. Dimethyl ether served as the reagent gas and was added to increase the pressure to $1-2 \times 10^{-5}$ torr (uncorrected). Helium buffer gas was added to bring up the total pressure into the millitorr regime. With the exception of freeze-pump-thaw cycles to remove noncondensible gases, all analytes were used as received from Aldrich Chemical Company (Milwaukee, WI). Labeled compounds were synthesized from the corresponding acid and the perdeutero alcohol via esterification [23]. Dimethyl ether- $d_{6}$, obtained from Isotec (Miamisburg, OH), was used to generate $[\mathrm{M}+$ $\left.\mathrm{CD}_{3}\right]^{+}$ions in the ITMS. Prior to collisional activation, the appropriate reagent ion of dimethyl ether was isolated and permitted to react with the analyte for a time sufficient to maximize the desired ion current. The ion of interest was isolated by using the apex isolation method [24] and subjected to CAD. Typical collisional activation conditions consisted of excitation of the ion of interest at its resonant frequency at $200-450 \mathrm{mV}$ for $10 \mathrm{~ms}$.

Semiempirical molecular orbital calculations were done by using MOPAC 6.0 [25] on a DEC Alpha AXP 4000/620 (Digital Equipment Corp., Maynard, MA). All geometries were optimized by using the AM1 hamiltonian [26, 27] and analytical derivatives of the energy. The ground state structures were obtained by minimization of chemically reasonable structures.

\section{DISCUSSION}

The structures of the compounds used in this study are shown in Figure 1. An array of substrates that possess one or two acid or ester functionalities and varying rigidity were chosen to allow a complete systematic study of functional group interactions. A simple comparison of the reactivity of fumaric and maleic acids would not provide significant insight into the role of functional group interactions because the influence of 
<smiles>C/C(=C\C(=O)O)C(=O)O</smiles><smiles>O=C(O)/C=C\C(=O)O</smiles>

Mesaconic acid

Fumaric acid<smiles>C=CC(=O)O</smiles><smiles>C=C(C)C(=O)O</smiles>

Acrylic acid

Methacrylic acid<smiles>O=C(O)CCC(=O)O</smiles><smiles>CC(CC(=O)O)C(=O)O</smiles>

Succinic acid

Methyl succinic acid<smiles>CCC(=O)O</smiles><smiles>COC(=O)/C=C/C(=O)OC</smiles>

Propionic acid

$$
\text { Dimedhyl }
$$
fumarate<smiles>CCC(=O)OC</smiles><smiles>C=CC(=O)OC</smiles>
Methyl
propionatc

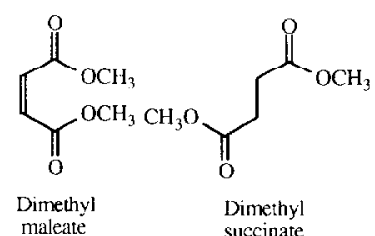<smiles>C=C(C)C(=O)OC</smiles>
Methyl
methacrylaie

Figure 1. Molecules examined in this study.

the olefin is overlooked. It is possible that the olefin would constrain the geometry of the molecule to such a degree that the results from this rigid system would not be applicable to the more pervasive flexible systems. Therefore succinic acid, the flexible analog of maleic acid, also was examined. In addition, the chemistry of acrylic acid, chosen to model "half" of a fumaric acid molecule, was compared with that of propionic acid. Methacrylic acid, with its extra electron-releasing methyl group, was chosen to examine the effect of olefin substitution on reactivity. Fragmentation of the corresponding esters would provide insight into the role of the acid protons and the effect of methyl substitutions. Monomethyl succinate allows a competition between the dehydration and methanol elimination pathways so that the dominating functional group can be cvaluated.

\section{Collision-Activated Dissociation Results}

As a method to probe the influence of carboxylate group interactions, collison-activated dissociation spectra were recorded for each $[\mathrm{M}+\mathrm{H}]^{+}$and $[\mathrm{M}+15]^{+}$ ion. These spectra are listed in Tables 1-4. All of the protonated acids, with the exception of methacrylic acid, primarily dehydrate after collisional aclivation, as shown in Table 1. Protonated methacrylic acid eliminates carbon monoxide instead. Harrison [28] postulated a rearrangement to account for this loss, which is facilitated by the ability of the vinyl methyl to stabilize the cationic charge at the tertiary carbon.

Table 1. CAD of protonated acids ${ }^{\mathrm{a}}$

\begin{tabular}{lrcc}
\hline Products & \multicolumn{3}{c}{ Neutral lost on CAD (\%) } \\
\cline { 2 - 4 }$[\mathrm{M}+\mathrm{H}]^{+}$ & $\mathrm{H}_{2} \mathrm{O}$ & $\mathrm{CO}$ & $\left(\mathrm{H}_{2} \mathrm{O}+\mathrm{CO}\right)$ \\
\hline \hline Fumaric acid & 100 & 0 & 0 \\
Maleic acid & 100 & 0 & 0 \\
Succinic acid & 100 & 0 & 0 \\
Mesaconic acid & 50 & 0 & 100 \\
Citraconic acid & 100 & 0 & 0 \\
Methyl succinic acid & 100 & 0 & 15 \\
Acrylic acid & 100 & 0 & 0 \\
Methcrylic acid & 0 & 100 & 0 \\
Propionic acid & 100 & 0 & 0 \\
\hline
\end{tabular}

all values are rounded to the nearest $5 \%$

Collision-activated dissociation of the $[M+15]^{+}$ products of the acids, shown in Table 2, results in either dehydration (maleic, succinic, citraconic, and methylsuccinic acids) or elimination of methanol (fumaric, mesaconic, and propionic acids), again with the exception of methacrylic acid, which undergoes decarbonylation. The general trend for the tendency toward dehydration or loss of methanol can be rationalized as follows. Fumaric acid, mesaconic acid, and the monoacids cannot lose water from their methylated products because there is no feasible intramolecular source for the essential second proton. Therefore, methanol is directly cleaved from the methylated portion of the molecule. This loss is likely preceded by a 1,3-proton migration from the hydroxyl group to the methoxyl. For the diacids in which the two groups can interact, proton migration is facile and therefore the elimination of water is favored.

Table 3 shows that nearly all the protonated esters lose methanol upon CAD, as expected. Protonated monomethyl succinate proved to be an exception: it lost water instead. This result can be rationalized by using the Hammond postulate [29], which states that the transition states of endothermic reactions are more similar to the products than to the reactants. Therefore, if an ion has two endothermic dissociation pathways

Table 2. CAD of methylated acids ${ }^{a}$

\begin{tabular}{lrrr}
\hline \multirow{2}{*}{$\begin{array}{l}\text { Product ion } \\
{[\mathrm{M}+15]^{+}}\end{array}$} & \multicolumn{3}{c}{ Neutrals lost on CAD (\%) } \\
\cline { 2 - 4 } & $\mathrm{H}_{2} \mathrm{O}$ & $\mathrm{MeOH}$ & $\mathrm{CO}$ \\
\hline \hline Fumaric acid & 0 & 100 & 0 \\
Maleic acid & 100 & 0 & 0 \\
Succinic acid & 100 & 0 & 0 \\
Citraconic acid $_{\text {Mesaconic acid }}$ & 100 & 0 & 0 \\
Methyl succinic acid $_{\text {Acrylic acid }}$ & 15 & 100 & 0 \\
Methacrylic acid & 0 & 0 & 0 \\
Propionic acid & 0 & 100 & 0 \\
\hline
\end{tabular}

a All values are rounded to the nearest $5 \%$.

Peaks not listed: $m / z$ 99, 20\%; $m / z \quad 85,15 \%$. 
Table 3. CAD of protonated esters ${ }^{a}$

\begin{tabular}{lrrrrr}
\hline \multirow{2}{*}{$\begin{array}{l}\text { Product ion } \\
{[\mathrm{M}+\mathrm{H}]^{+}}\end{array}$} & $\mathrm{MeOH}$ & $(\mathrm{MeOH}+\mathrm{CO})$ & $\mathrm{H}_{2} \mathrm{O}$ & $\left(\mathrm{H}_{2} \mathrm{O}+\mathrm{CO}\right)$ & $\mathrm{CO}$ \\
\cline { 2 - 6 } & 100 & 20 & 0 & 0 & 0 \\
\hline \hline Dimethyl fumarate & 100 & 0 & 0 & 0 & 0 \\
Dimethyl maleate & 100 & 30 & 0 & 0 & 0 \\
Dimethyl succinate & 100 & 0 & 0 & 0 & 0 \\
Methyl propionate & 100 & 0 & 0 & 0 & 0 \\
Methyl acrylate & 0 & 0 & 0 & 0 & 100 \\
Methyl methacrylate & 0 & 0 & 100 & 15 & 0 \\
Monomethyl succinate & 0 & 0 & & & 0
\end{tabular}

${ }^{a}$ All values are rounded to the nearest $5 \%$.

with similar transition states, one can approximate the difference between the two critical energies by examination of the relative stabilities of the products. It has been demonstrated that the gradual heating of ions during collisional activation [30] in the quadrupole ion trap yields product ions that result from kinetic control of the fragmentation rather than thermochemical control; therefore the products are determined by the critical energies. The relative energies of the fragmentation products of the $[\mathrm{M}+\mathrm{H}]^{+}$of monomethyl succinate can be calculated, as shown below (this argument was suggested by a reviewer):

First, the competing eliminations of water and methanol can be written

$$
\begin{aligned}
& \text { (monomethyl succinate }+\mathrm{H})^{+} \\
& \quad \rightarrow(\text { succinic anhydride }+\mathrm{H})^{+}+\mathrm{CH}_{3} \mathrm{OH} \\
& (\text { monomethyl succinate }+\mathrm{H})^{+} \\
& \quad \rightarrow\left(\text { succinic anhydride }+\mathrm{CH}_{3}\right)^{+}+\mathrm{H}_{2} \mathrm{O}
\end{aligned}
$$

The enthalpic relationships can be written

$$
\begin{aligned}
\Delta H_{1}^{\circ}= & \Delta H_{f}^{\circ}(\text { succinic anhydride }+\mathrm{H})^{+} \\
& +\Delta H_{f}^{\circ}\left(\mathrm{CH}_{3} \mathrm{OH}\right) \\
& -\Delta H_{f}^{\circ}(\text { monomethyl succinate }+\mathrm{H})^{+} \\
\Delta H_{2}^{\circ}= & \Delta H_{f}^{\circ}\left(\text { succinic anhydride }+\mathrm{CH}_{3}\right)^{+} \\
& +\Delta H_{f}^{\circ}\left(\mathrm{H}_{2} \mathrm{O}\right) \\
& -\Delta H_{f}^{\circ}(\text { monomethyl succinate }+\mathrm{H})^{+}
\end{aligned}
$$

It is known that

$$
\begin{gathered}
\left.\Delta H_{f}^{\circ} \text { (succinic anhydride }+\mathrm{H}^{+}\right) \\
=\Delta H_{f}^{\circ}\left(\mathrm{H}^{+}\right)+\Delta H_{f}^{\circ} \text { (succinic anhydride) } \\
-\mathrm{l} \text { 'A (succinic anhydride) } \\
\Delta H_{f}^{\circ}\left(\text { succinic anhydride }+\mathrm{CH}_{3}^{+}\right) \\
=\Delta H_{f}^{\circ}\left(\mathrm{CH}_{3}^{+}\right)+\Delta H_{f}^{\circ} \text { (succinic anhydride) } \\
-\mathrm{MCA}(\text { succinic anhydride) } \\
(\mathrm{MCA}=\text { methyl cation affinity) }
\end{gathered}
$$

so the following relationship can be derived

$$
\begin{aligned}
\Delta H_{1}^{\circ}-\Delta H_{2}^{\circ}= & \Delta H_{f}^{\circ}\left(H^{+}\right)-\Delta H_{f}^{\circ}\left(\mathrm{CH}_{3}^{+}\right) \\
& +\Delta H_{f}^{\circ}\left(\mathrm{CH}_{3} \mathrm{OH}\right)-\Delta H_{f}^{\circ}\left(\mathrm{H}_{2} \mathrm{O}\right) \\
& + \text { MCA (succinic anhydride) } \\
& - \text { PA (succinic anhydride) }
\end{aligned}
$$

The first few terms have known values and can be combined to yield $114 \mathrm{kcal} /$ mole:

$$
\begin{aligned}
= & 114-[\text { PA (succinic anhydride) } \\
& - \text { MCA (succinic anhydride) }]
\end{aligned}
$$

Table 4. CAD of methylated esters ${ }^{\mathrm{a}}$

\begin{tabular}{lrcccc}
\hline Product ion & \multicolumn{5}{c}{ Neutrals lost (\%) } \\
\cline { 2 - 6 }$[\mathrm{M}+15]^{\dagger}$ & $\mathrm{CO}$ & $\mathrm{CH}_{3} \mathrm{OCH}_{3}$ & $(\mathrm{MeOH}+\mathrm{CO})$ & $\left(\mathrm{CH}_{3} \mathrm{OCH}_{3}+\mathrm{CO}\right)$ & $\mathrm{MeOH}$ \\
\hline \hline Dimethyl fumarate & 30 & 100 & 30 & 60 & 0 \\
Dimethyl maleate & 30 & 100 & 0 & 25 & 0 \\
Dimethyl succinate & 0 & 0 & 100 & 0 & 5 \\
Methyt propionate & 0 & 100 & 0 & 0 & 0 \\
Methyl acrylate & 0 & 100 & 0 & 0 & 0 \\
Methyl methacrylate & 0 & 40 & 15 & 0 & 15 \\
Monomethyl succinate & 0 & 0 & 0 & 0 & 100 \\
\hline
\end{tabular}

a All values are rounded to the nearest $5 \%$

${ }^{b}$ Other ions: $m / z$ 59, $100 \%$ 
If [PA (succinic anhydride) - MCA (succinic anhydride)] $<114 \mathrm{kcal} / \mathrm{mol}$, dehydration is preferred. By using the bracketing method, the methyl cation affinity of succinic anhydride was determined to be greater than that of dimethyl ether, whereas its proton affinity was less than that of cyclopentanone. Therefore, by approximating $\Delta S_{\mathrm{rx} \|} \approx 0$, the methyl cation affinity of succinic anhydride is $>95 \mathrm{kcal} / \mathrm{mol}$ [31] and its proton affinity is < $198.8 \mathrm{kcal} / \mathrm{mol}$ [32]; thus water loss is kinetically favored over methanol elimination for protonated monomethyl succinate.

Table 4 summarizes the CAD results for the $[\mathrm{M}+$ $15]^{+}$products of the esters. Most of these $[M+15]^{+}$ ions lose dimethyl ether, with the only exceptions being dimethyl succinate and monomethyl succinate. The $[\mathrm{M}+15]^{+}$ion of dimethyl succinate dissociates by loss of methanol with carbon monoxide, and the $[\mathrm{M}+15]^{+}$ion of monomethyl succinate eliminates methanol exclusively. In addition, the $[\mathrm{M}+15]^{+}$ions of dimethyl fumarate and dimethyl maleate show loss of carbon monoxide. For dimethyl succinate the unusual loss of methanol and carbon monoxide is attributed to the increased acidity of the $\alpha$-protons after methylation of the carbonyl uxygen. The flexibility of dimethyl succinate allows the removal of one of these protons, as shown in Figure 2. The transition state for elimination of methanol is both entropically and kinetically more favorable than the tight transition state required for the methyl transfer that leads to dimethyl ether loss. The loss of carbon monoxide from the [M+ $15]^{+}$ions of dimethyl fumarate is postulated to occur through the pathway shown in Figure 3. A similar mechanism can be devised for the $\mathrm{CO}$ elimination from the analogous ions of dimethyl maleate.

The $[\mathrm{M}+15]^{+}$of monomethyl succinate eliminates methanol exclusively and, aside from the mechanism outlined in Figure 2, two others can be postulated. The first possibility is that methylation occurs at the hydroxyl oxygen, and then loss of methanol follows as a direct cleavage. This pathway was disproven by generation and collisional activation of the $\left[\mathrm{M}+\mathrm{CD}_{3}\right]^{+}$ product formed by ion-molecule reactions with perdeuterated dimethyl ether ions. Equal amounts of methanol- $d_{0}$ and methanol- $d_{3}$ are eliminated, yet only methanol- $d_{3}$ would be lost if methylation at the hydroxyl oxygen and cleavage occurred. It seems reasonable that the neutral monomethyl succinate should have an intramolecular hydrogen bond, just like maleic acid, and this hydrogen bond would direct electrophilic addition to the acid carbonyl group. Therefore, the second possibility to account for the observed

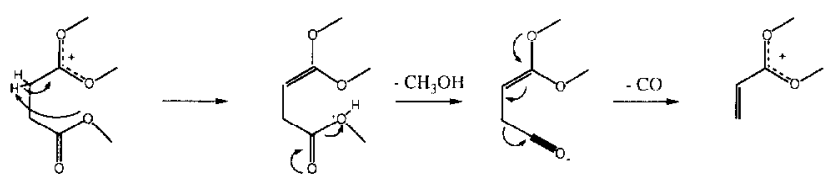

Figure 2. Pustulated mechanism for formation of $[M+15]^{+}$of dimethyl succinate and mechanism for methanol elimination.

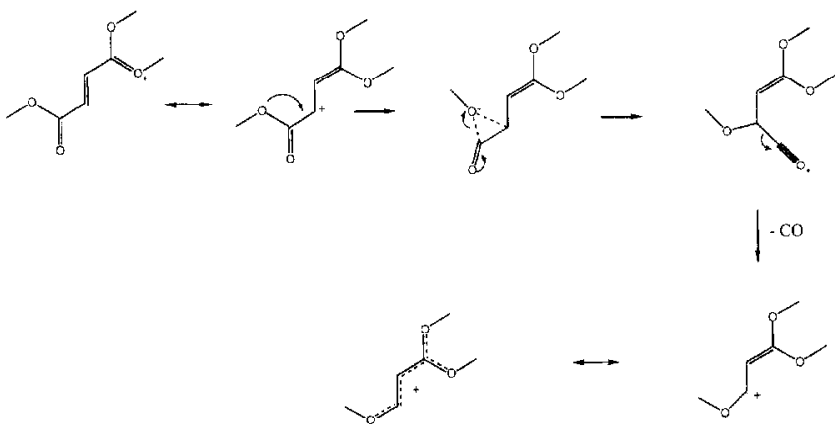

Figure 3. Postulated mechanism for carbon monoxide elimination from $[M+15]^{+}$of dimethyl fumarate.

elimination is shown in Figure 4. This $[\mathrm{M}+15]^{+}$ion formed has an intramolecular hydrogen bond, and after the appropriate bond rotations, this proton can be easily removed by either methoxy group, which permits facile methanol elimination.

The neutral losses observed for the dissociation of the $[\mathrm{M}+15]^{+}$of monomethyl succinate may proceed through a transition state similar to that observed for the $[\mathrm{M}+15]^{+}$ion of monomethyl maleate- $d_{3}$ (which loses $\mathrm{CD}_{3} \mathrm{OH}$ and $\mathrm{CH}_{3} \mathrm{OH}$ in a 1:1 ratio, just like the $\left[\mathrm{M}+\mathrm{CD}_{3}\right]^{+}$ion of monomethyl succinate). To test this hypothesis, monomethyl succinate- $d_{1}$ was synthesized with the deuterium on the acid functionality. The $[\mathrm{M}+15]^{+}$product for this deuterated compound was found to lose only $\mathrm{CH}_{3} \mathrm{OD}$ on collisional activation. Thus, the proton lost with the methanol originates on the hydroxy group of the acid, and the mechanism shown in Figure 4 is preferred.

The $[\mathrm{M}+15]^{+}$products of diesters will eliminate dimethyl ether unless the diester is flexible enough to permit proton abstraction for subsequent methanol loss. Similarly, the $[\mathrm{M}+15]^{+}$products of acids will eliminate methanol unless there is a labile proton available for dehydration, as in the case of $Z$-acids.
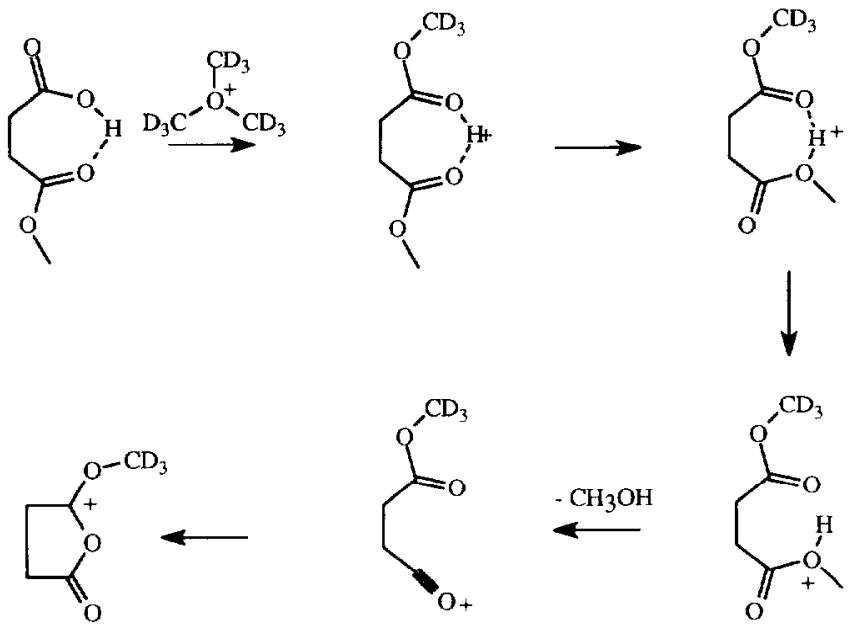

Figure 4. Postulated mechanism for formation of $[M+15]^{+}$of monomethyl succinate and mechanism for methanol elimination. 


\section{Site of Methylation}

To elucidate the site of methylation, AM1 calculations and a series of labeling experiments were undertaken. Dimethyl maleate was chosen as a general model for this study. AM1 calculations suggest that the methylation of dimelhyl maleale occurs on the carbonyl oxygen and that the structure is best represented with two equivalent methoxy groups bound to a carbocation, as in Figure $5 a$ and $b$. (The two structures for Figure $5 c$, which differ by only a single rotation, are included for comparison purposes only.) This methylation site agrees with previous studies that examined the methylation of esters (based on prior work on site of methylation of esters [33]). From this proposal, it is then possible to determine whether the dimethyl ether loss upon $\mathrm{CAD}$ of the $[\mathrm{M}+15]^{+}$ions occurs via a $1,3-$ methyl transfer or if it is a methyl transfer between functional groups. The reaction of dimethyl maleate with dimethyl ether- $d_{6}$ produced $\left[\mathrm{M}+\mathrm{CD}_{3}\right]^{+}$which was isolated prior to collisional activation. The three possibilities for methyl- $d_{3}$ transfer and their anticipated CAD products are shown in Figure 6. Figure 6a predicts that methylation of dimethyl maleate on one carbonyl oxygen followed by a 1,3-methyl transfer will lead to elimination of only dimethyl ether $-d_{3}$. The same loss is expected for the reaction pathway shown in Figure $6 \mathrm{~b}$, in which methylation occurs at the methoxy oxygen. If the representation that the methylated ester has two equivalent methoxy groups is correct and if the observed dimethyl ether loss occurs only by methyl transfer to the other functional group as shown in Figure 6c, one would expect (neglecting secondary kinetic isotope effects), an equal elimination of dimethyl ether- $d_{3}$ and dimethyl ether- $d_{0}$. In fact, on collisional activation, the ratio for the elimination of $\mathrm{Cl}_{3} \mathrm{OCD}_{3}$ to $\mathrm{CII}_{3} \mathrm{OCII}_{3}$ was observed to be $2: 1$, as shown in Figure 7a. This result suggests that the fragmentation reaction proceeds through pathway $6 \mathrm{c}$ with little or no contributions from pathways $6 a$ or $6 b$. However, two other reasonable possibilities remain: the presence of a secondary kinetic isotope effect or complete scrambling of the methyl groups prior to elimination.

To determine if either of these latter two factors was operative, dimethyl maleate- $d_{6}$, dimethyl fumarate- $d_{6}$, and dimethyl succinate- $d_{6}$ were synthesized. Colli-
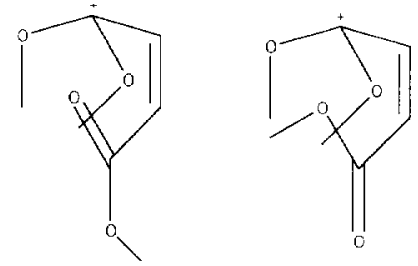

b

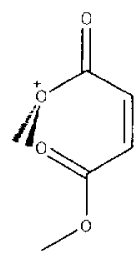

25.2 koalimale

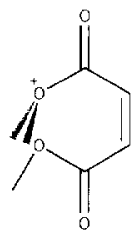

c 48.0 kcalimole
Figure 5. Maleic acid minima calculated by using AM1.

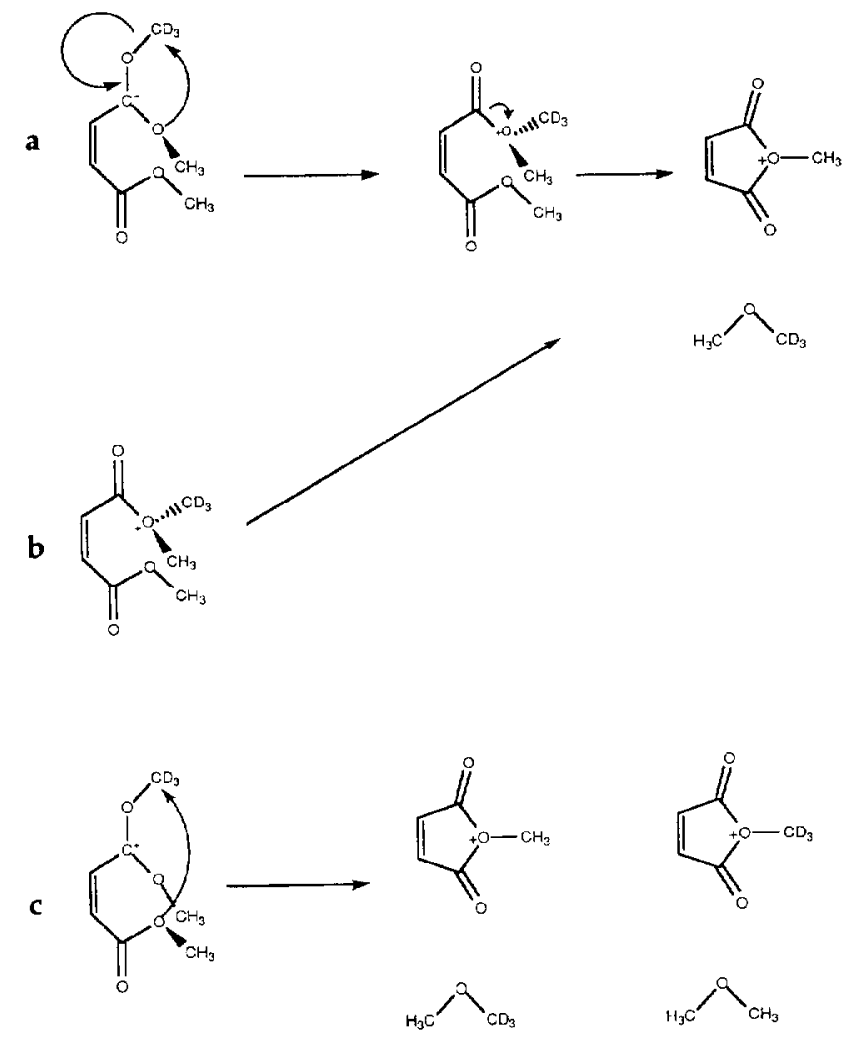

Figure 6. Possible routes for dimethyl ether elimination.
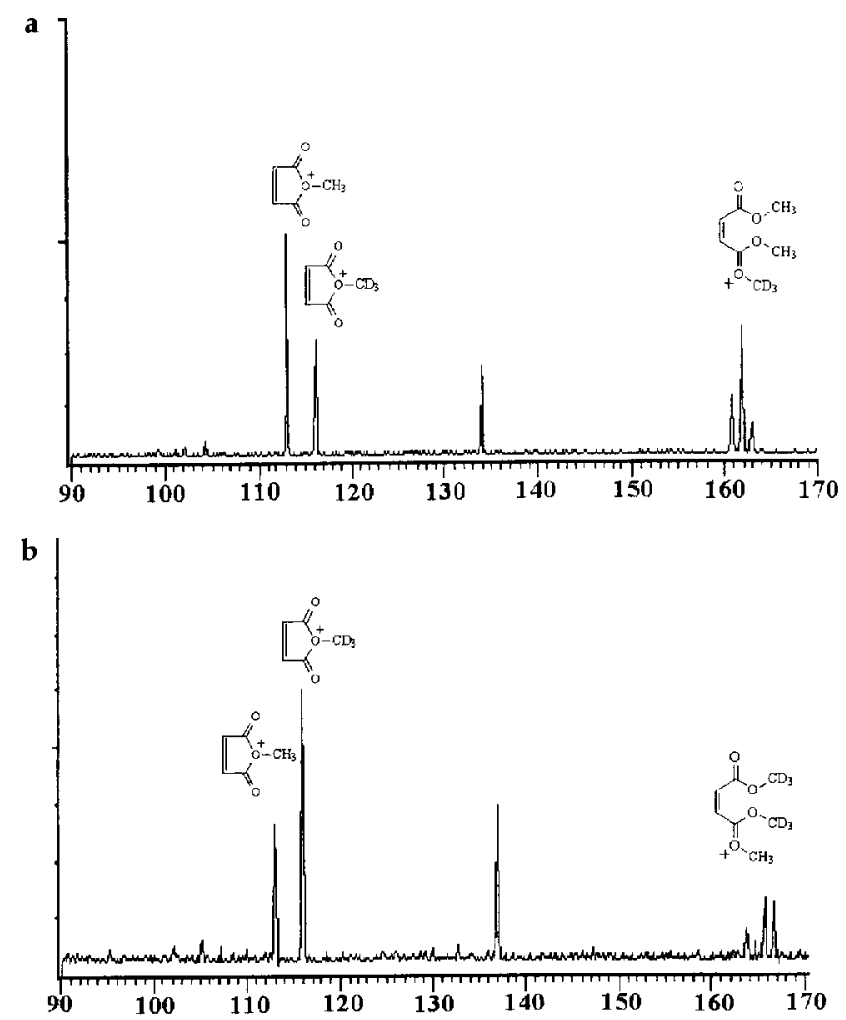

Figure 7. (a) $\mathrm{CAD}$ of $\left[\mathrm{M}+\mathrm{CD}_{3}\right]^{+}$of dimethyl maleate- $d_{0}$. (b) $\mathrm{CAD}$ of $\left[\mathrm{M}+\mathrm{CH}_{3}\right]^{+}$of dimethyl maleate- $d_{6}$. 
sional activation of the $[\mathrm{M}+15]^{+}$ion of dimethyl fumarate- $d_{6}$ exhibited only a loss of dimethyl ether- $d_{3}$, which suggests the dimethyl ether loss occurred via a 1,3-methyl transfer from the carbonyl oxygen of dimethyl fumarate to its methoxy oxygen. (To test if this product was actually the result of an ion-molecule reaction between the $[\mathrm{M}+15]^{+}$of dimethyl fumarate$d_{6}$ and neutral dimethyl fumarate- $d_{6}$, the pressure of the neutral dimethyl fumarate- $d_{6}$ was increased 1 order of magnitude and only a small amount of $\left[\mathrm{M}+15-\mathrm{CD}_{3} \mathrm{OCD}_{3}\right]^{+}$(relative to $[\mathrm{M}+15-$ $\left.\mathrm{CD}_{3} \mathrm{OCH}_{3}\right]^{+}$) was observed, which indicated that even at very high dimethyl fumarate pressures $\left(>1 \times 10^{-5}\right.$ torr), few ion-molecule reactions that result in a methyl transfer occur. Therefore, such reactions should contribute much less at $1 \times 10^{-6}$ torr dimethyl fumarate.) It seems unlikely that the initial site of methylation of dimethyl fumarate is on the methoxy oxygen because calculations show this site to be $\sim 22 \mathrm{kcal} / \mathrm{mol}$ less favorable than the carbonyl oxygen, and this pathway would disagree with the results for labeled dimethyl maleate.

Figure $7 \mathrm{~b}$ shows that the $[\mathrm{M}+15]^{+}$ion of dimethyl maleate- $d_{6}$ exhibits a $2: 1$ loss of dimethyl ether- $d_{3}$ to dimethyl ether- $d_{6}$ on collisional activation, which suggests complete scrambling of the methyls prior to the dimethyl ether elimination. Similarly, methylated dimethyl succinate- $d_{6}$ shows nearly statistical methyl scrambling prior to the loss of methanol, which provides further evidence that the two functional groups interact similarly to dimethyl maleate. Thus it appears that the slow step in the dimethyl ether elimination from the $[\mathrm{M}+15]^{+}$ions of the esters is a 1,6-methyl transfer, and that this elimination is preceded by a rapid interfunctional methyl exchange. From this series of experiments, it is clear that the $[\mathrm{M}+15]^{+}$ion of dimethyl maleate undergoes rapid scrambling of all the methyl groups and then eliminates dimethyl ether as shown in Figure 6c. Moreover, the pathways shown in Figure 6a and $b$, under the activation conditions uscd, contribute insignificantly, if at all, to this ion population.

Based on the foregoing results, methylation occurs at the carbonyl oxygen and is preceded, in the case of diesters, by an equilibration of methyl groups between interacting functional groups prior to dimethyl ether elimination.

\section{Conclusions}

By using a combination of CAD, thermochemical calculations, and labeled compounds, we have determined that the electrophilic addition of the methyl cation to the diacids and diesters occurs primarily on the carbonyl oxygen. For the methylated diesters, collisional activation results in a rapid interfunctional group methyl cation exchange (in those molecules with interacting functional groups) followed by the slow 1, 6methyl transfer that leads to loss of dimethyl ether.
This work also demonstrates that ion-molecule reactions can give insight into the reactive conformers of some neutrals. For example, a comparison of the CAD products of succinic acid with those of maleic and fumaric acids, readily shows that because the dissociation of the $[\mathrm{M}+15]^{+}$ion of succinic acid more closely resembles that of the $[M+15]^{\dagger}$ ion of maleic acid, the succinic acid likely adopts a cisoid geometry either prior to or during the fragmentation reaclion. This similarity is echoed by the fragmentation pattern of the $[\mathrm{M}+15]^{+}$ions of dimethyl succinate $d_{6}$.

\section{Acknowledgments}

This work was funded by the National Science Foundation, the National Institute of Health, the Welch Foundation, the Dreyfus Foundation and the Sloan Foundation. We thank Dr. Jean-Claude Tabet for helpful discussions.

\section{References}

1. Benoit, F.; Holmes, J. L.; Isaacs, N. S. Org. Mass Spectrom. $1969,2,591$.

2. Weinkam, R. J. I. Am. Chem. Soc. 1974, 96, 1032.

3. Fenselau, C. C., Robinson, C. H. I. Am. Chem. Soc. 1971, 93, 3070 .

4. Brodbelt, J. S.; Wysocki, V. H.; Cooks, R. G. Org. Mass Spectrom. 1988, 23, 54.

5. Eichmann, E. S.; Brodbelt, J. S. I. Am. Soc. Mass Spectrom. 1993, 4, 230

6. Eichmann, E. S.; Brodbelt, J. S. Org. Mass Spectrom. 1993, 28, 655.

7. Eichmann, E. S.; Brodbelt, J. S. Org. Mass Spectrom. 1993, 28, 737.

8. Respondek, J. Schwarz, H; Van Gaever, F.; Van der Sande, C. C. Org. Mass Spectrom. 1978, 13,618.

9. Harrisun, A. G., Rao, K. M.; Kallury, R. Org. Mass Spectrom. $1980,15,277$.

10. Wysocki, V. H., Burinsky, D. I., Cooks, R. G. I. Org. Chem. $1985,50,1287$.

11. Bowie, J. H.; Williams, D. H.; Madsen, P.; Schroll, G.; Lawesson, S.-O. Tetrahedron 1967, 23, 305.

12. Benoit, F.; Holmes, J. L. Org. Mass Spectrom. 1972, 6, 541.

13. Fales, H. M; Milne, G. W. A.; Nicholson, R. S. Anal. Chem. 1971, 43, 1785.

14. Weinkam, R. J.; Gal, J. Org. Mass Spectrom. 1976, 11, 188.

15. Iajima, S.; Ishiguro, A.; Tobita, S. Int. I. Mass Spectron. Ion Processes 1987, 75, 147.

16. Dallinga, I. W.; Nibbering, N. M. M.; van der Greef, J.; Ten Noever de Brauw, M. C. Org. Mass Spectrom. 1984, 19, 10.

17. Vainiotalo, P.; Malkonen, P. J. Org. Mass Spectrom. 1987, 22, 688.

18. Several extensive reviews on functional group interactions and on cis-trans diacids-diesters: (a) Vairamani, M.; Saraswathi, M. Mass Spectrom. Rev. 1992, 10, 491; (b) Mandelbaum, A. Mass Spectrom. Rev. 1983, 2, 223; (c) Vainiotalo, P.; Harrison, P. J. Org. Mass Spectrom. 1987, 22, 687; (d) Splitter, J. S.; Turecek, F. Application of Mass Spectrometry to Organic Stereochmistry; VCH Publishers: New York, 1991.

19. Harrison, A. G.; Nacson, S.; Mandelbaum, A. Org. Mass Spectrom. 1987, 22, 283.

20. Weisz, A.; Mandelbaum, A.; Shabinowitz, J.; Hunt, D. F. Org. Mass Spectrom. 1984, 19, 238.

21. Brodbelt, J.; Liou, J.; Donovan, T. Anal. Chem. 1991, 63, 1205.

22. Burrows, E. P. Org. Mass Spectrom. 1992, 27, 763. 
23. Pavia, D. L.; Lampman, G. M.; Kriz, G. S., Jr. Introduction to Organic Laboratory Techniques: A Contemporary Approach; W. B. Saunders: Philadelphia, 1976; p 94.

24. Weber-Grabau, M.; Kelley, P. E.; Syka, J. E. P.; Bradshaw, S. C.; Brodbelt, J. S. Proceedings of the 35th Annual Conference of the American Society for Mass Spectrometry; 1987; $p 1114$.

25. Stewart, J. P. QCPE 455. MOPAC 6.0 (QCPE 455) is available from the Quantum Chemistry Program Exchange, Indiana University, Bloomington, IN.

26. Dewar, M. J. S.; Storch, D. M. I. Am. Chem. Soc. 1985, 107, 3898.

27. Dewar, M. J. S. Org. Mass Spectrom. 1993, 28, 305.

28. Harrison, A. G.; Ichikawa, H. Org. Mass Spectrom. 1980, 15, 244.
29. Isaacs, N. S. Physical Organic Chemistry; Wiley: New York, 1987; p 104

30. Brodbelt, J. S.; Wysocki, V. H.; Cooks, R. G. Org. Mass Spectrom. 1988, 23, 54.

31. Szulejko, J. E.; Fisher, J. J.; McMahon, T. B.; Wronka, J. Int. J. Mass Spectom. Ion Processes 1988, 83, 147.

32. Lias, S. G.; I iehman, J. F.; Levin, R. D. J. Phys. Chem. Ref. Data 1984, 13, 695.

33. (a) Audier, H. E.; Berthomieu, D.; Hudhomme, P.; Monteiro, C.; Mourgues, P. Org. Mass Spectrom. 1990, 25, 87; (b) Morizur, J.-P.; Martigny, I.; Tortajada, J.; Geribaldi, S. Org. Mass Spectron. 1990 25, 89. 